\title{
ESTUdO DAS HABILIDADES DE MEMÓRIA E RACIOCÍNIO SIMBÓLICO E NÃO- SIMBÓLICO DE CRIANÇAS E ADOLESCENTES SURDAS POR MEIO DA BATERIA padrão do Universal Nonverbal Intelligence Test ${ }^{1}$
}

\author{
Study OF MEMORY AND SYMBOLIC AND NON-SYMBOLIC REASONING SKILLS OF \\ DEAF CHILDREN AND ADOLESCENTS USING STANDARD BATTERY OF UNIVERSAL \\ N OnVerbal Intelligence TEST
}

\section{Cláudia Furtado BORGES² \\ Renata Ferrarez Fernandes LOPES 3 \\ EderaldoJoséLOPES 4}

\begin{abstract}
RESU M O : este estudo explorou as habilidades de memória eraciocínio simbólico enão simbólico de crianças e adolescentes com deficiência auditiva através das análises de desempenho da Bateria Padrão do Universal N onverbal Intelligence Test (UNIT). Participaram deste estudo 55 crianças e adolescentes surdos. Os dados coletados foram anal isados através de anál ises paramétricas. Os dados mostram que a memória simbólica, a capacidadedemanipular erepresentar a informação simbólica memorizada está sendo mel horada por atividades escolares e/ ou extracurriculares.
\end{abstract}

PALAVRAS-CHAVE: educação especial; inteligência; surdez; habilidades cognitivas.

ABSTRACT: this study aimed to explore memory and symbolic and non symbolic reasoning skills of deaf children and adolescents using the analyses of performance of the standard battery of Universal Nonverbal IntelligenceTest (UNIT). Fifty five participants took part in thestudy. The collected data wereanalyzed through parametric statistics. The data suggest that the capacity to manipulate and to represent memorized symbolic information may beenhanced by school and/ or extracurricular activities.

KEYWORD S: Special Education; Intelligence; Deafness; Cognitive A bilities.

\section{InTRODUÇÃO}

A inteligência éum dos constructos mais controvertidos em Psicologia. A confusão resideem questões tais como: Devemos considerar a inteligência como culturalmente definida ou livre de cultura? A inteligência deve ser considerada como uma única aptidão ou como muitas? Ela está ligada à rapidez cognitiva? Como ela pode ser avaliada em termos neurológicos e psicológicos?

\footnotetext{
${ }^{1}$ Nós agradecemos à Fundação de Amparo à Pesquisa do Estado de Minas Gerais (FAPEMIG) pelo apoio financeiro recebido (Proc. SHA 1940/ 06)

2 Mestranda em Psicologia pela UFU - MG. kksempsi@hotmail.com

${ }^{3}$ Professora Adjunta do Instituto de Psicologia da Universidade Federal de Uberlândia - MG. rfernandeslopes@fapsi.ufu.br

${ }^{4}$ Professor Associado do Instituto de Psicologia da Universidade Federal de Uberlândia - Uberlândia MG. ederaldol@umuarama.ufu.br
} 
Para a maioria dos psicólogos a inteligência é a capacidade para o comportamento adaptável e orientado a um objetivo (MEYERS, 1998). Alguns pesquisadores argumentam que o comportamento inteligente traduz-se na habilidade de uma pessoa ser capaz de adaptar-se com sucesso às exigências da escola ou do trabalho. O comportamento inteligente parece, em alguns casos, relacionar-se à cultura, especial mente no que tange aos eventos-meta que exigem comportamento verbal. Por outro lado, outros pesquisadores insistem que a inteligência é a habilidade de resolver todos os tipos de problemas independentemente da cultura.

No que diz respeito às influências ambientais, muitos estudos comparam crianças que têm uma rica estimulação ambiental com crianças que têm uma pobre estimulação ambiental ou culturalmente diferentes, sugerindo que as experiências prévias influenciam sobremaneira o desempenho em testes de inteligência (COHEN, 1969).

No contexto da surdez, a dificuldade de comunicação é uma das manifestações mais evidentes da deficiência auditiva, desencadeando dificuldades do surdo para conviver einteragir numa sociedade oral. Muitas referências ligadas à pesquisa acerca da educação dos surdos apontam para os problemas de comunicação como um dos fatores que se relacionam com a crença de comprometimento intelectual ecognitivo. Estudos queinvestigam a relação destas variáveis são raros, tanto na literatura internacional quanto nacional. Também são raros instrumentos de aval iação psicológica e cognitiva adaptados para avaliar estetipo de população.

Os testes psicológicos são medidas padronizadas de comportamentos ligados às capacidades mentais ou deaspectos da personal idade. Todo instrumento de avaliação psicológica deve ser válido (o teste medeo que se propôs a medir) e fidedigno (a precisão do teste quanto ao constructo que pretende medir).

O Universal N onverbal Intelligence Test (UNIT) (BRACKEN; MCCALLUM, 1998) éum teste de intel igência que provêuma medida geral (fator g) da inteligência e de habilidades cognitivas efoi delineado para ser usado com crianças eadolescentes com idades variando de 5 a 17 anos.

O UNIT é o único teste multidimensional de QI que pode ser administrado de forma não verbal, embora o aplicador tenha a liberdadedemanter o rapport com a criança ou com os adolescentes, desde que esta conversa não se refira aos subtestes do UN IT, garantindo, assim, a administração não verbal do teste. Isto favorece a população em geral e, especial mente, aquel es indivíduos que não poderiam ser aval iados (aval iação da inteligência) com testes verbais, em função das diferenças culturais e de natividade (crianças eadolescentes educados em sua língua natal como, por exemplo, comunidades de chineses, de hispânicos, de al emães), bem como crianças com problemas de linguagem. 
A o contrário de muitos testes não verbais, que são compostos principal mente por matrizes, o UNIT éestruturado por subtestes que estimulam o envolvimento da criança na tarefa, al ém de exigir do examinando diferentes tipos de respostas como: manipulações de fichas, utilização de papel e lápis e/ ou até mesmo indicação de uma resposta. Todos os estímulos que compõem os subtestes são universais emulticulturais, eas tarefas são projetadas para serem envolventes para qualquer criança independente da raça, etnia e cultura (BRACKEN; MCCALLUM, 1998).

O testefoi estruturado a partir do modelo hierárquico de inteligência com habilidades gerais, sendo composto por seis subtestes: memória simbólica, desenho do cubo, memória espacial, raciocínio analógico, memória para objetos e labirintos. Sua administração é flexível, uma vez que pode-se aplicar a bateria abreviada, com apenas 2 subtestes, a fim de se proceder a um screening da função intelectual; a bateria padrão, com 4 subtestes, para uma tomada de decisão diagnóstica e a bateria extensa, com todos os subtestes, para uma avaliação diagnóstica mais profunda.

É importante ressaltar que o UNIT possui uma estrutura em componentes de memória e raciocínio (escalas primárias) e simbólicas e não simbólicas (escal as secundárias). Nas escalas primárias, memória eraciocínio são construtos fundamentais na medida de inteligência. As tarefas de memória (habilidades associativas) são compostas por transformação e correlação com e entre o(s) estímulo(s) de entrada - input. As tarefas de raciocínio (habilidades cognitivas) exigem transformação do estímulo de entrada - input-, através de planejamento e/ ou avaliação (BRACKEN ; McCALLUM , 1998).

Lopes et al . (2006) salientaram que

[...] outros testes avaliam a memória de curto prazo através de tarefas mnemôni cas deformato serial simples (por exemplo, memória para palavras, span de dígitos), bem como apresentam uma mediação verbal no quetangeà aplicação dos itens e às respostas da criança, o UNIT toca em habilidades associativas mais amplas à medida queapresenta os estímul os deforma visual para o sujeito do teste (exigindo muitas vezes uma mediação simbólica envolvendo rotulação, organização ecategorização) e exige respostas motoras (por exemplo, rearranjo físico decartões deresposta), ampliando a sensibilidade de mensuração desta habilidade associativa. O raciocínio é considerado o "coração" da inteligência, pois requer do indivíduo habilidades de planejamento eaval iação baseados na dedução de relações.

Já as escalas secundárias do UNIT descrevem processos mentais subjacentes que facilitam o desempenho da criança na mediação simbólica (envolvendo representação, analogia e conceitualização) e na mediação não simbólica (envolvendo abstração e processamento não verbal), sendo estes processos mentai s aspectos subordinados às escal as primárias (LOPES et al., 2006). 
O testetambém permite, através de linhas guias que se encontram no manual, estabelecer hipóteses interpretativas a partir da comparação entre a subescalas dos subtestes. Por exemplo, a comparação das escalas de raciocínio e dememória permite ao examinador investigar seo raciocínio não verbal émelhor desenvolvido do quea memória de curto prazo; sea habilidade de síntese, análise ereorganização do estímulo émel hor quea habilidade decompreender ereproduzir um estímulo; sea habilidade para se concentrar durante as atividades de solucionar um problema é melhor do que a habilidade para se atentar a detalhes visuais relevantes.

Assim, o UNIT parece ser um instrumento de avaliação psicológica que abrange um conjunto de características psicométricas que o tornam um instrumento interessante para avaliar crianças e adolescentes portadores de deficiência auditiva.

As características psicométricas estabelecidas pela equipe de pesquisadores por meio de análise fatorial em dados preliminares indicou a presença de 1 fator (Q -FSI- fator g) que explica 90, 01 \% da variância total e coeficiente de fidedignidade al pha $=0,936$. Em conjunto, os dados obtidos até $o$ presente mostram a viabilidade de aplicação deste teste não verbal e revela um grande potencial para avaliar intelectual menteamostras brasileiras com diferentes características, se configurando numa al ternativa para os testes intelectuais verbais conhecidos até o presente.

Para avaliar as habilidades e as competências de pessoas portadoras dedeficiência auditiva, especialmente decrianças ejovens, muitas vezes é preciso transcender a simples aplicação de um instrumento de aval iação de uma lista de testes recomendados. Randy Kamphaus, em um deseus livros dirigido a psicólogos e psicopedagogos intitulado "A valiação clínica da inteligência das crianças: Um manual para a prática profissional" apresentou a seguinteafirmação:

[...] O examinador que não tem familiaridade com crianças portadoras de deficiência auditiva ou com impedimento auditivo no geral podeser capaz de obter escores detestee eventualmente obter escores acurados. A questão central, entretanto, éa interpretação de escores detestes deinteligência eo planejamento do tratamento da criança. Um examinador expert ( porém sem impedimento auditivo) pode ter dificuldade em entender melhor a etiologia, o curso e os tratamentos do problema da criança, embora entenda muito bem de testagem psicológica. Esta éuma questão similar a visitar um psiquiatra para tratar de problemas cardiovasculares. Enquanto o psiquiatra pode obter escores de um el etrocardiograma e outros testes, eu pessoalmente me sentiria melhor nas mãos de um cardiologista (KAM PHAUS, 1993, p. 400).

Revisando 208 estudos sobre inteligência em portadores de surdez, Braden (1994) concluiu que o QI das pessoas surdas é substancialmente menor para testes deestruturação verbal ou para testes cujos procedimentos deaplicação são baseados na linguagem verbal. 
Qual quer aval iação intelectual de pessoas com déficits auditivos deve ser realizada levando-se em consideração o significado mais amplo de processos comunicativos envolvendo conteúdo, método, atitude e emoções, qualidade, quantidade e eficácia da comunicação.

Dessa forma, o UNIT é um dos poucos testes inteiramente não verbal, tanto da perspectiva de sua estruturação (itens que compõem os subtestes) quanto de sua aplicação, permitindo, portanto, uma avaliação da intel igência surdos da forma mais ecológica, considerando os impedimentos relacionados a esta necessidade especial, uma vez que os testes tradicionais raramente trazem normatizações para estetipo de população especial.

A ssim, o objetivo desteestudo foi fazer uma exploração das habilidades dememória eraciocínio simbólico enão-simbólico de crianças eadolescentes surdos em 4subtestes do UNIT (memória simbólica, memória espacial, raciocínio analógico e desenho do cubo) que compõem a bateria padrão deste instrumento não-verbal de avaliação da inteligência. A análise destas habilidades cognitivas contribuiu para compreender melhor os componentes cognitivos relacionados à inteligência nesta amostra.

\section{MÉTOdo}

\subsection{Participantes}

A amostra foi constituída de 55 crianças e adolescentes surdos com idades entre 5 e 17 anos com diferentes graus de deficiência auditiva, sendo todas frequentadoras de instituições na cidade de Uberlândia e Patos de Minas. As instituições eram de ensino regular ou instituições que ofereciam atividades extraescolares (música, aula de Libras, suporte pedagógico, etc).

\subsection{I nStRUMENTOS}

Foi utilizado o Teste de Inteligência N ão Verbal (UNIT) (BRACKEN; MCCALLUM, 1998). É um instrumento que avalia habilidade de raciocínio e memória simbólica e não simbólica. O seu o kit de aplicação contém: um dos livrosestímulo (livro 1) onde se encontram os quatro subtestes que permitem aferir habilidades cognitivas de interesse deste estudo: memória simbólica e espacial, desenho do cubo e raciocínio analógico, nove cubos bicolores (verde-branco), suporte para colocação (arranjo) dos cubos, oito fichas verdes e oito fichas pretas, papel cartonado contendo uma grade $3 \times 3$ e outra $4 \times 4$, dispostas uma de cada lado do papel cartonado para realização do subteste de memória espacial, dez fichas contendo material simbólico (fichas apresentando individualmente figuras da seguinte série desímbolos universais: bebê, garota, garoto, mulher ehomem (cinco cor verdee cinco na cor preta) e cronômetro. 
De acordo com Cunha (2000), é interessante num processo de avaliação a utilização de outras ferramentas complementares. Neste sentido, foi utilizado um questionário de perguntas fechadas, que foi enviado aos pais junto com o termo deconsentimento, a fim de verificar informações referentes à etiologia, nível de perda auditiva, idade e escolaridade tanto da criança como dos pais.

\subsection{Procedimentos}

A pós a aprovação do Comitê de Ética e com a autorização da instituição, foi enviada uma correspondência aos pais (anmenese) com o objetivo de explicar a proposta do projeto e obter informações sobre alguns dados da criança e do seu meio social e familiar. O dia, o local e o horário de aplicação foram previamente acordados entre a instituição e os pesquisadores.

Cabe destacar que indivíduos que têm dificuldades na comunicação em função de problemas de aprendizagem ligadas à linguagem expressiva ou receptiva, como é o caso dos deficientes auditivos, apresentam desafios únicos para os pesquisadores (VERN ON, 1965). Desta forma, só examinadores supertreinados tanto na aplicação do testequanto no domínio de LIBRAS puderam aplicar o teste, a fim de estabelecer um bom rapport com o examinando.

\subsubsection{A plicação do teste}

A aplicação do teste foi feita individualmente numa sala diferente daquela em queo participante desenvolvia suas atividades de aprendizagem. Ela foi precedida por um rapport conduzido com os participantes, feito em LIBRAS ou numa linguagem acessível a eles, por pesquisadores treinados tanto na língua de sinais quanto na execução do teste. A pós informar aos participantes sobreo objetivo da aval iação, toda a comunicação passou a ser real izada através dos sinais universais preconizadas no manual do UNIT. Um exemplo destes sinais universais pode ser observado na Figura 1.

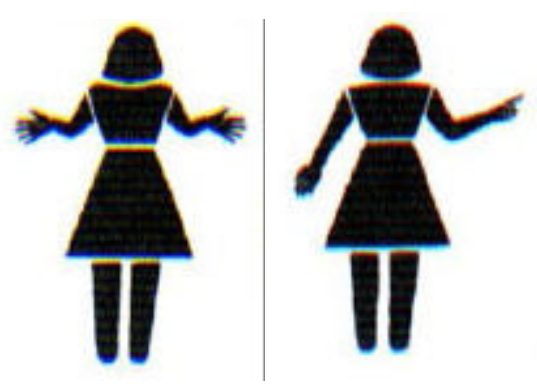

Figura 1 - Exemplos de comuni cação não verbal realizada durante a aplicação do UNIT. N a figura à esquerda, o sinal significa qual éa resposta?. N a figura à direita, o sinal significa agora você!. 
O teste foi aplicado seguindo a ordem, como traz o manual (BRACKEN ; M CCA LLUM, 1998): memória simbólica, memória espacial, desenho de cubo e memória simbólica. O tempo de aplicação do teste durou por vol ta de 30 a 40 minutos, com intervalos entre os subtestes se o participante achasse necessário. Ao final da aplicação, o participante era encaminhado novamente para sua sala de origem.

\section{Resultados}

\subsection{ESCRIÇÃo GERAL DA AMOSTRA}

A análise preliminar dos dados foi realizada a partir do protocolo de 55 crianças e adol escentes com deficiência auditiva, nos mais variados níveis de comprometimento. N as seções seguintes será apresentado um perfil da amostra estudada em função de al gumas características que a literatura da área destaca e que podem interferir nas habilidades intelectuais e cognitivas deste tipo de população (MACEDO, 2005; MARCHESI, 1995; POKER, 1995; VERNON, 1965). Deve-se ressal tar que al guns dados são categorizados como "não responderam". Isto deve-se ao fato de que alguns pais ou responsáveis dos participantes que fizeram parte do nosso banco de dados não responderam a ficha de anamnese.

A amostra foi constituida de 20 participantes do sexo masculino (36,4\%) e 35 participantes do sexo feminino (63,5\%). A idadedos participantes variou de 7 a 17 anos, com média de 14,04 (DP =2,64).

Em relação à escolaridade, a amostra variou entre a 1a série do ensino fundamental ea $2^{a}$ série do ensino médio. Este tipo de subagrupamento (baseado na escolaridade) indicou que a mi noria dos aval iados cursava a 3 a série do ensino fundamental (3,6\%) ea maioria, com 18,2\%, encontrava-sematriculada na 4ấ série.

Foi investigado em qual ouvido o examinando possuía a deficiência e o nível decomprometimento da mesma. Observou-seque $54,5 \%$ da amostra possuia deficiência auditiva nos dois ouvidos, sendo que 40,0\% da amostra apresentavam comprometimento severo. Em relação ao tipo de comunicação do examinando, $1,8 \%$ da amostra utilizavam a oral ização (método utilizado para ensi nar o surdo a falar), leitura labial (técnica em quepalavras emitidas pelo interlocutor são captadas pela interpretação dos movimentos dos lábios) e uma comunicação própria (denominada para o tipo de comunicação com gestos sem referência na Linguagem de Sinais). A lém disso, 18,2\% da amostra utilizavam a LIBRAS. Pode-se perceber também que $20 \%$ comunicavam-se razoavelmente com a LIBRAS; $18,2 \%$ dominavam a oral ização; $20 \%$ possuiam uma leitura labial razoavelmente boa; contudo, 20\% não dominavam a leitura labial.

Em relação ao exercício de atividade extra, 34,5\% da amostra faziam algum ti po deatividade extraescolar como: aula de música, aula de reforço, libras, capoeira, etc. Foram avaliadas, em relação aos pais, as categorias presença ou 
ausência de deficiência auditiva. Observou-se que a maioria dos pais $(56,4 \%)$ e das mães $(60,0 \%)$ eram ouvinte.

Ao fazer uma análise descritiva da amostra, observou-se que, dependendo do tipo deagrupamento dos dados (variáveis idadeeescolaridade), as amostras tornavam-se mais ou menos heterogêneas. Isto quer dizer que se a amostra fosse agrupada por escol aridade, seria criada uma condição de análiseem queos subgrupos eram muito mais heterogêneos. Por exemplo, na 1ª sériedo ensino fundamental havia examinandos de 7 a 17 anos. Por outro lado, quando esses examinandos eram agrupados por idade, a heterogeneidade era menor.

Devido ao tamanho e, sobretudo, à heterogeneidade da amostra estudada, não éfácil tirar conclusões gerais dos model os cognitivos propostos para os deficientes auditivos. Grande parte dos estudos sobre memória eraciocínio na população com essa deficiência partedo pressuposto dequeos deficientes auditivos compõem um grupo rel ativamente homogêneo, semel hanteà população ouvinte, o que uma análise preliminar dos dados não confirma. Com base nesta descrição da amostra citada acima, observa-sea existência de diferenças individuais dentro do grupo desurdos. Pode-sefalar em subgrupos diferenciad os a partir das seguintes variáveis: o nível de perda auditiva, a idade do início deficiência, sua etiologia eos fatores educacionais e comunicativos. Estas variáveis são as mesmas que influem na evolução cognitiva dos deficientes auditivos. Por essa razão, as variáveis nível de perda auditiva, fatores educacionais e comunicativos serão testadas numa próxima etapa do trabalho. De imediato, o que se pode concluir é que qualquer afirmação general ista sobre processos cognitivos envolvendo memória eraciocínio desurdos é contra-indicada.

\subsection{A nálises PARAMÉtricas}

A utilização deanálises paramétricas para os dadosfoi viável, a despeito do número de participantes da amostra, pois a anál ise de assimetria dos dados indicou valores de?² superiores a 0,24 (MILES; SHEVLIN , 2001).

\subsubsection{Análises de correlações}

Foram calculadas as médias aritméticas, os desvios padrão e os coeficientes de correl ação entre as variáveis do estudo por meio da correlação de Pearson. Para a análise da matriz de correlação, considerou-se a seguinte classificação de magnitude dos coefi cientes de correlação: 0,10 - 0,29: fraca; 0,300,49: moderada e 0,50 ou maior: forte.

Foram obti das as seguintes correlações entre os subtestes de memória simbólica eidade $(r=0,332, p<0,05)$; entre o subteste desenho de cubo eidade ( $r$ $=0,297, p<0,05)$; e entre o subteste memória espacial eidade $(r=0,291, p<0,05)$ (Tabela 1). 
Tabela 1 - Médias aritméticas, desvios padrão e coeficientes de correlação ( $r$ de Pearson) entre idade os subtestes do UNIT.

\begin{tabular}{llllllll}
\hline Variáveis & Média & Desvio Padrão & $\mathbf{1}$ & $\mathbf{2}$ & $\mathbf{3}$ & $\mathbf{4}$ & $\mathbf{5}$ \\
\hline 1 - Idade & 14,04 & 2,63 & & & & & \\
2- Memória Simbólica & 6,71 & 3,34 & $0,332^{*}$ & & & & \\
3 - Desenho de Cubo & 22,27 & 8,73 & $0,297^{*}$ & 0,516 & & & \\
4- Memória Espacial & 10,75 & 3,97 & $0,291^{*}$ & 0,433 & 0,533 & & \\
5- Raciocínio Analógico & 7,84 & 3,19 & 0,025 & 0,247 & 0,534 & 0,423 \\
\hline
\end{tabular}

Já nas correlações entre escolaridade e os subtestes do UNIT (Tabela 2), observou-seuma única correlação significativa, quefoi entrememória simbólica eescolaridade, tendo sua intensidade moderada $(r=0,368 ; p<0,01)$.

Tabela 2 - Médias fatoriais, desvios padrões e coeficientes de correlação ( $r$ de Pearson) entre escolaridade os subtestes do UNIT.

\begin{tabular}{|c|c|c|c|c|c|c|c|}
\hline Variáveis & Média & Desvio Padrão & 1 & 2 & 3 & 4 & 5 \\
\hline 1 - Escolaridade & 5,91 & 2,75 & & & & & \\
\hline 2-Memória Simbólica & 6,71 & 3,34 & $0,368^{*}$ & & & & \\
\hline 3 - Desenho de Cubo & 22,27 & 8,73 & 0,192 & 0,516 & & & \\
\hline 4-Memória Espacial & 10,75 & 3,97 & 0,189 & 0,433 & 0,533 & & \\
\hline 5 - Raciocínio Analógico & 7,84 & 3,19 & 0,032 & 0,247 & 0,534 & 0,423 & \\
\hline
\end{tabular}

\subsubsection{A NÁLISE DE VARIÂNCIA}

A fim deverificar se havia diferença entre os subgrupos que compõem a amostra, submeteram-seos escores obtidos nos subtestes do UNIT a uma AN OVA a um fator para a variável escolaridadee outra para a idade.

A ANOVA confirmou que os oito subgrupos testados ( $11^{a}, 2^{a}$ e $3^{a}$ séries; $4^{a}$ série; $5^{\underline{a}}$ série; $6^{a}$ série; $7^{a}$ série; $8^{\underline{a}}$ série do ensino fundamental; $1^{\circ}$ a ano do ensino médio e 20 ano do ensino médio) diferem apenas no desempenho quanto à variável memória simbólica[ $F(7,47)=2,13, p<0,05]$. Houvediferenças significativas

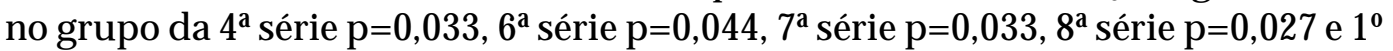
colegial $p=0,021$.

Entretanto, quando se submeteram os escores obtidos nos subtestes do UNIT a uma ANOVA com a variável idade, os resultados mostraram que houve diferença significativa entreos grupos quanto ao subteste desenho do cubo $[F(6,48)$ $=3,08, p<0,05]$. Os dados mostram uma diferença estatisticamentesignificativa no grupo com 10 - 11 anos se comparada com os participantes do grupo de 15 anos. 


\section{D ISCUSSÃo}

A utilização do UNIT deu-se pelo fato dessetesteatingir uma extensão mais ampla na avaliação defatores relacionados às variáveis que envolvem o fator g como: planejamento, raciocínio espacial, solução de problemas, organização, dentre outros (LUBIN SKI, 2004), além deser um instrumento não verbal que pode anular ou neutralizar variáveis culturais e sociais, que muitas vezes são influenciáveis na interpretação.

Considerando os dados expostos eanalisando-os em conjunto, percebese que eles apontam para a variável memória simbólica como tendo um papel preponderantena explicação dos resultados. Esta convergência pareceindicar que a capacidade de mani pular e representar a informação si mbólica memorizada está sendo enfatizada no processo de estimulação acadêmica e/ ou não-acadêmica nesta amostra.

Capovilla (2001) ressal ta quea educação dos surdos vem deum enfoque na oralização, tornando o nível deescolarização do surdo muito inferior em relação aos ouvintes. Tal movimento começou a mudar a partir de 1980 quando a Libras ganhou força na sociedade ouvinte, pois se passou a reconhecer a importância de aprender a língua de sinais, de modo que sua inserção passou a ser obrigatória nos diversos contextos sociais: tel evisão, escolas, empresas dentre outras. Além disso, o processo de aprendizagem da língua de sinais requer memorização ecorrelação entre imagens de objetos, semântica, contextual ização esi nai s. N o mesmo sentido, Marchesi (1995) aponta que o desenvolvimento cognitivo do surdo está diretamente relacionado ao processo de estimulação nos ambientes (escola, família, dentre outros) ena forma pela qual as tarefas são ensinadas aos surdos. Deve-seconsiderar a Libras não apenas como um meio de comunicação, mas como uma ferramenta de desenvolvimento para outros fatores como: 1) os códigos de memória; 2) os processos deleitura; 3) o el emento mediador einclusi vo nas relações interpessoais. Isto sugere que, possivel mente, a estimulação desta amostra leva em conta o treino de habilidades como atenção a detalhes, percepção de significado, mediação simbólica everbal, memória visual, formação de conceitos e organização perceptual. A lém disso, parece que o processo de escolarização tem enfatizado habilidades de memória verbal/ simbólica mais do quehabilidades de raciocínio, o que podelevar a considerações equivocadas sobreo nível intelectual de deficientes auditivos. Se a avaliação intelectual enfatizar aspectos do raciocínio, então o desempenho do avaliando será prejudicado, pelo menos é o que se observa na análise preliminar desses dados.

Outro fator relevante, é a opinião dos examinandos sobre o rapport, o teste e sua aplicação com a finalidade de verificar se houve alguma dificuldade encontrada, seja em rel ação à compreensão da tarefa ou em relação a sua execução. Todos el es afirmaram não ter encontrado dificul dade em compreender as tarefas ou mesmo em executá-las. Muitos inicial mente acreditavam que iriam responder perguntas ou fazer atividades "chatas". Relataram também que sentiam que 
estavam "jogando" ou "brincando" e que o instrumento mais interessante foi o subtestememória espacial.

\section{CONCLUSÕES}

Este trabalho possui algumas características pioneiras que vão ao encontro das propostas de estudos que envolvem a reestruturação de fatores psicométricos na avaliação de pessoas surdas. N esse sentido, devem-seconsiderar os poucos estudos que se preocupam com uma avaliação mais qualitativa do que quantitativa, com o objetivo de minimizar variáveis que possam levar a interpretações errôneas e limitadoras do potencial cognitivo das pessoas com deficiência auditiva.

Embora os estudos sobreas propriedades psicométricas do UNIT para a população em geral sejam incipientes no Brasil, estudos preliminares como o de Lopes et al . (2006), assi m como o relatado nesteartigo, apontam para a riqueza de fatores ligados à memória e ao raciocínio que podem ser extraídos de análises qualitativas desse teste com pessoas surdas. Isto não minimiza a importância de novos estudos, com amostras maiores, a fim de se trabal har com um instrumento válido efidedigno para essetipo de amostra.

Finalmente, os resultados deste estudo podem ser um norteador e estimulador para a compreensão das habilidades cognitivas das pessoas surdas. Podeser prejudicial "discriminar" ou "minimizar" variáveis (etiologia da perda, nível de perda, escolarização) quepodem estar diretamente correlacionadas com a constituição, o desenvolvimento ea operacional ização destas habilidades nos mais diversos contextos. Além disso, é preciso entender que muitas pessoas com deficiência auditiva podem estar impossibilitadas de exercitar suas habilidades por ter um ambiente que não oferece condições adaptativas para el as (p. ex., por falta de comunicação) ou estimulantes (p. ex., por falta de capacitação dos profissionais da educação) que atendam as suas necessidades e possam ampliar seu potencial deaprendizagem.

\section{ReferênCIAS}

BRACKEN, B. A.; MCCALLUM, R. S. The U niversal N onverbal Intelligence Test. Chicago: RiversidePublishing Company, 1998.

BRADEN, J. P. D eafness, deprivation, and IQ . N ew York: Plenum, 1994.

CA POVILLA, F. C. D icionário en cicl opédico ilustrado trilingueda língua desinais brasileira. São Paulo: EDUSP, 2001.

COHEN, A.R. Conceptual styles, culture conflict, and nonverbal test of intelligence. A merican A nthropologist, Arlington, v.71, n.5, p. 828-856, 1969.

CUNHA, JA. Psicodiagnóstico V. Porto A legre: Artes Médicas, 2000. 
KAMPHAUS, R. W. Clinical assessment of children's intelligence. Needham Heights. MA: Allyn \& Bacon, 1993.

LOPES, R, F. F., etal. Características psicométricas da bateria padrão do universal nonverbal intelligencetest (UNIT): um estudo preliminar. Psicologia Escolar eEducacional, Campinas, v. 10, n. 2, p. 273-282, 2006.

LUBINSKI, D. Introduction to the special section on cognitive abilities: 100 Years After Spearman's (1904) 'General Intelligence', objectively determined and measured. Journal of Personal ity and Social Psychology, Washington, v. 86, n. 1, p. 96-111, 2004.

MACEDO, M.F. Educação escol ar para pessoas com surdez: dos primórdios aos tempos atuais. São Paulo: Mimeo, 2005.

MARCHESI, Á. El desarollo cognitivo y lingüístico de los ninos sordos. Madrid: Alianza Psicologia, 1995.

MEYERS, D. Introdução à psicologia geral. Rio de Janeiro: LTC, 1998.

MILES, J.; SHEVLIN, M. A pplying regression \& correlation: A guide for students and researchers. London: SA GE Publications, 2001.

POKER, R. B. T roca simbólica edesenvolvimento cognitivo em crian ças su rdas: uma proposta de intervenção educacional. 1995. Dissertação deM estrado (Educação) - FaculdadedeFilosofia e Ciências da UNESP, Marília, 1995.

VERNON, P. E. A bility factors and environmental influences. A merican Psychologist, Washington, v. 20, n. 9, p. 723-733, 1965. 